\title{
Removing Speckle Noise in Synthetic Aperture Radar Images using Combined Intensity Coherence Vector \& Hybrid Filtering
}

\author{
Dr.D.Suresh,Professor ${ }^{1 *}$, Dr.D.Ramesh ${ }^{2}$, Dr.S.Satheesbabu ${ }^{3}$, Dr.S,K.Somasundaram ${ }^{4}$ \\ ${ }^{1}$ Professor, Department of CSE,PSNA College of Engineering and Technology,Dindigul \\ ${ }^{2}$ Assistant Professor,Department of CSE,Anna University,University College of Engineering,Dindigul \\ ${ }^{3}$ Associate Professor,Department of CSE,PSNA College of Engineering and Technology Dindigul. \\ ${ }^{4}$ Assistant Professor, (Selection Grade), Department of Information, Technology, PSG College of Technology, Coimbatore, \\ Tamil Nadu, India
}

\begin{abstract}
Noise will be unavoidable in image securing practice and denoising is a fundamental advance to recoup the image quality. The image of Synthetic Aperture Radar (SAR) is intrinsically misrepresented in dot noise that happens because of coherent nature of the dispersing wonders. Denoising SAR images target eliminating dot while safeguarding image highlights, for example, surface, edges, and point targets. The blend of nonlocal gathering and changed area filtering has coordinated the cutting edge denoising methods. Notwithstanding, this methodology makes an intense suspicion that image fix itself gives a brilliant guess on the genuine boundary, which prompts predisposition issue transcendently under genuine dot noise. Another impediment is that the for the most part utilized fix pre-determination techniques can't productively avoid the exceptions and harm the edges. The SAR image is infused with spot noise, and afterward edge based marker controlled watershed division is applied to recognize the homogeneous locales in SAR image. For every locale, the local pixels are distinguished by utilizing Intensity Coherence Vector (ICV) and are denoised autonomously by utilizing a half and half filtering, which contains the improved forms of ice, middle and mean channel. The exploratory outcomes show that the proposed strategy outflanks different techniques, for example, fix based filtering, non-nearby methods, wavelets and old style dot channels in wording higher wavelets signal-to-noise and edge conservation proportions relatively.
\end{abstract}

Article Received: 20 September 2020, Revised: 30 November 2020, Accepted: 18 December 2020

\section{Introduction}

The Synthetic Aperture Radar (SAR) imaging is more sensible for different environment conditions [1] than the optical far off recognizing. Regardless, the significant issue in SAR imaging is the proximity of recognize, a sign ward granular commotion, which adulterates the quality and examination of the picture. Subsequently, it is basic to decrease the spots from the SAR pictures before the picture assessment starts, at the same time, the picture nuances, for instance, target centers, surfaces, shapes should not be get disturbed. All through the latest a very long time there is different assessment responsibility on despeckling, as the new time of SAR imaging has typically gains the experts towards this issue. The possibility of despeckling computation is immovably related with Additive White Gaussian Noise (AWGN) denoising. Regardless, the spot commotion is multiplicative in nature; the log-change is applied on the signs to change over them into added substance to fit the approaches with included substance noise removing procedure. There are three classes of despeckling counts (I) spatial, (ii) wavelet-based, and (iii) nonlocal sifting.

The spatial removing procedures take a little piece and replace the target pixel of the window by strategies for a weighted typical. For a low-pass channel, where the heaps are proportionate, reduces the commotion just as upsets the edges, structures and surfaces. Considering the various view of a comparable sign is one response for this issue, and the heaps should be evaluated reliant on quantifiable models of sign and commotion and, the local estimations of the picture.

\section{Related work}

Various filtering calculations have been proposed over the most recent quite a few years for SAR images spot denoising (Lee et al. 1994), the greater part of which depend on the insights of pixels and their relationship with their environmental factors (Lee, 1981, Deledalle et al. 2009, Parrilli et al, 2012). What's more, pixels even in one image can follow disparate measurable scatterings, which acquire entanglements assessing boundaries (Cheng et al. 2013). Conversely, Lie et al (2013) presented an elective route for despeckling, which smothers spot without displaying the dot straightforwardly. The despeckling calculations can be grouped into two classifications: the principal classification takes L various looks of SAR images of a similar scene, and normal them to decrease dot noise. Notwithstanding, it likewise lessens ground goal of the image with respect to the quantity of looks. The second class of approaches works with single image of a scene with dot filtering calculations both in spatial and recurrence space (Gagnon and Jouan, 1997; Espinoza Molina et al., 2012; Iqbal et al., 2013). This paper centers around the primary classification. The traditional channels (Lee, 1980, 1986; Frost et al., 1982; Kuan et al., 1985; Lee et al., 1994) can be utilized to denoise SAR images in spatial space. These channels work by partitioning the SAR images into sub-windows, and for each sub-window the inside pixel is supplanted dependent on the 
consistency of the pixels accessible in the sub-window. These methodologies can deliver better denoising in static images. In the event of SAR images they either safeguard dot noise or harm the frail signals at heterogeneous regions, for example, edge, point targets or surface region. The gamma-MAP channel (Lopes et al., 1990) and the refined gamma-MAP channel (Baraldi and Parmiggiani, 1995) consider both the spot model and the reflectivity likelihood thickness capacities. The gamma-MAP channel profoundly relies upon the portrayal of the gamma esteem. In any case, the decisions of neighborhood window size and direction have more prominent effect on the exhibition of these spatial channels. The greater part of these channels utilize a neighborhood investigation window with fixed size and shape, albeit movable windows of these calculations for nearby spatial varieties are required. Yu and Action (2002) proposed a Speckle Reduction Anisotropic Diffusion (SRAD) channel to despeckle the SAR images just as to save image edges better.

\section{Proposed system}

The filtering technique proposed in this work which has two stages. It begins by isolating the SAR image into number of homogeneous districts, and for every area nearby mean channel is applied to denoise the SAR image. The homogeneous locales are distinguished by utilizing an improved marker controlled watershed division calculation and the coherent pixels are related to the measure called Intensity Coherence Vector (ICV).

\subsection{Intensity Coherence Vector}

Naturally, the pixel's intensity coherence is characterized as how much pixels of that intensity are individuals from huge likewise hued areas. These noteworthy districts are alluded as coherent areas, and it is seen that they are huge in depicting pictures. The rationality measure ICV describes pixels as either sound or muddled. Reasonable pixels are a bit of some sizable circumscribing region, while incomprehensible pixels are unquestionably not. A concealing lucidness vector addresses this game plan for each concealing in the picture. ICV's keep cognizant pixels in a solitary picture from organizing incongruous pixels in another. This grants fine capabilities that can't be made with histograms. This property of ICV is used here to recognize the local soundness pixels at each sub-region got from improved watershed segmentation calculation. The accompanying content clarifies the calculation of ICV measure.

\section{Computing ICV's}

The initial phase in processing ICV is like the calculation of a histogram. At first the image is discretized into 'nc' number of unmistakable powers to kill the little motions between neighboring pixels. The following stage is to arrange the pixels inside a given region as either intelligent or incomprehensible. An intelligible pixel is a bit of an enormous social event of pixels of a comparable concealing, while a disjointed pixel isn't. The pixel bunch is controlled by registering associated parts. An associated part $\mathrm{C}$ is a greatest arrangement of pixels so that for any two pixels $\mathrm{p}$, $\mathrm{p}^{\prime} \in \mathrm{C}$, there is a way in $\mathrm{C}$ among $\mathrm{p}$ and $\mathrm{p}^{\prime}$. (Officially, a way in $C$ is a succession of pixels $p=p 1, p 2, \ldots, p n=p^{\prime}$ with the end goal that every pixel pi is in $\mathrm{C}$ and any two consecutive pixels pi, pi+1 are nearby one another. Two pixels are viewed as contiguous on the off chance that one pixel is among the eight nearest neighbors of the other. As such, slanting neighbors are incorporated.) Note that associated parts are processed inside a given discretized intensity container. This viably portions the sub-district dependent on the discretized space.

Associated segments can be figured in a direct time. At the point when this is finished, every pixel will have a place with precisely one associated part. At that point the pixels are delegated either coherent or incoherent relying upon the size in pixels of its associated part. A pixel is coherent if the size of its associated part surpasses a fixed worth ' $t$ '; Otherwise, the pixel is incoherent. For a given discretized intensity territory, a portion of the pixels with that intensity will be coherent and some will be incoherent. Let the quantity of coherent pixels of the jth discretized shading $j$ and the quantity of incoherent pixels $\mathrm{j}$, and the all out number of pixels with that shading is $\mathrm{j}+\mathrm{j}$.

\section{a. Coherence Despeckling}

When the ICVs are recognized for every area, the district is set apart with the coherence name. Each name is intended for comparing coherence pixels. The pixels having a place with one specific mark is extricated and their intensity esteems are gotten from the first grayscale SAR image to locate their mean qualities. The coherence mark area has the base of $30 \%$ of pixels (Pass et al., 1997). Among the whole pixels in district alone is considered for denoising,

$\square \mathrm{j} / \mathrm{nr} \square 0.3$

Where $\square \mathrm{j}$, is the quantity of pixels with the coherence mark ' $\mathrm{j}$ ' and $\mathrm{nr}$ is the complete number of pixels accessible in the locale. At that point the every pixel's intensity esteem is supplanted with the determined mean worth, which is characterized as.

$\operatorname{Ii}(\mathrm{x}, \mathrm{y})=\square \operatorname{Ii}(\mathrm{x}, \mathrm{y}) / \mathrm{nr}$, where $\operatorname{Ii}(\mathrm{x}, \mathrm{y}) \square \mathrm{ci}$,

Where, ci is the coherence marks of the district ' $r$ ', Ii(x,y) is the intensity esteems from the SAR images has the comparing coherence name ci.

This system will be rehashed for outstanding coherence names accessible for the current area. Furthermore, the denoising is proceeded with the following locale, till the whole image is denoised.

The accompanying calculation sums up the proposed despeckling calculation

Step 1. Read as SAR image (If it is a shading image than convert it to grayscale). 
Step 2. Find the edge extents of the standardized SAR image by utilizing 'watchful' administrator

Step 3. Apply the watershed calculation anxious guide to get shut shapes.

Step 4. Discretize the image into 'nc' shading receptacles.

Step 5. Map the edge limits with the grayscale image to separate the portioned regions.

Step 6. For each divided district

a. Find the Intensity Coherence Vectors and name them

b. For every coherence name

c. Apply half and half filtering

Step 7. Output the denoised SAR image.

The presentation of the proposed filtering approach is contrasted and other existing denoising approaches and is depicted in the accompanying segment.

\section{FMM HYBRID FILTERING}

There is another technique of separating called cross breed channel, used to mix more than one channels together to procure the potential gain of all. Shanthi and Valarmathi (2011) proposed a count using Hybrid solicitation experiences channel HMM (Hybrid Mean Median) is proposed, which is a mix of mean and center channels to smother speck clamor in SAR concealing pictures. The introduction estimations shows that the proposed channel performs better similar to PSNR and MSE. Bhateja et al., (2014) proposed an improved neighborhood estimations channel for removing the spot commotion from the SAR pictures. The proposed channel is a blend of mean and crossbreed center channels, using a novel $7 \times 7$ sifting design. The display of the proposed channel is attempted against the standard Hybrid Median channels for which the surveyed characteristics show better acts with respect to PSNR and SSI. Furthermore, this channel has a novelistic property of killing commotion, near to edge upkeep and nuances protection. Zheng et al., (2014) proposed an independent change recognizable proof system by using k-infers gathering on the joined difference picture. This procedure uses the close by consistency of the qualification picture in the wake of using the mean channel and the edge information insurance of the differentiation picture in the wake of using the center channel for better difference picture depiction.

The standard idea of this paper is to cross breeds these channels to take all of their central focuses while the issues of each channel could be settled by the advantages of another. For the hybridization the improved types of mean, center and ice channels, for instance, Edge based Adaptive
Mean (EAM) channel (Shamsoddini and Trinder, 2012), Local Adaptive Median (LAM) channel (Qiu et al., 2004) and the Adaptive Frost (AF) channel (Zhihua and Zengguo, 2015) are used. Consequently the hybridization channel is Linear, Adaptive, Preserve the edges, fitting for spot commotion and moreover smoothen the pictures.

\section{EXPERIMENTS AND RESULTS}

In this work, the SAR images of mimicked and genuine are utilized to dissect the exhibition of the proposed SAR denoising strategy. At first, the reenacted images are corrupted by infusing dot noise, hence, the first images are treated as the genuine qualities and are utilized as mathematical measures to evaluate the presentation. In this test, five different techniques (i.e., Frost channel, PPB, LMMSE-LMMSE, and BM3D, BNL channel calculations) are chosen to contrast and the proposed strategy. The determination of these strategies depends on both accessibility of the codes and their importance to this work.

\subsection{SYNTHETIC IMAGES DATABASE}

An assortment of picture sources are considered in this testing, including the benchmark test picture, Lena, Barbara, Boats, and Lake. A unnaturally spotted image is produced by a sans image of noises with dot noise. L-look adequacy spotted images of size $512 \times 512$ upset by created dot noise is taken for tests, and the presentation of calculations is assessed by PSNR. The proposed calculation was actualized in MatLab® condition.

\subsection{REAL SAR IMAGES DATABASE}

To approve the adequacy of proposed calculation, the genuine SAR images got from four distinct storehouses (ALOS-PALSAR, Sentinel-1, Terra SAR, and AirSAR) has been utilized. Table 1 sums up the datasets utilized for assessment. The depiction and the information download of each dataset could be alluded in the given connection.

Table 1. Summary of Real SAR Image Datasets

\begin{tabular}{|l|l|l|l|l|}
\hline Dataset & Band & Type & $\begin{array}{l}\text { \#0f } \\
\text { Images }\end{array}$ & URLs \\
\hline $\begin{array}{l}\text { ALOS- } \\
\text { PALSAR }\end{array}$ & L & GeoTiff & 25 & $\begin{array}{l}\text { www.asf.alaska.edu/sar- } \\
\text { data/palsar/ }\end{array}$ \\
\hline Sentinel-1 & C & GeoTiff & 25 & scihub.esa.int/dhus/ \\
\hline Terra SAR & X & GeoTiff & 25 & terrasar-x-archive.infoterra.de/ \\
\hline AirSAR & L \& C & GeoTiff & 25 & airsar.jpl.nasa.gov \\
\hline
\end{tabular}




\subsection{PERFORMANCE EVALUATION}

The performance assessment of channels is a significant and essential issue on SAR picture despeckling. For manufactured spot pictures, the Peak Signal-to-Noise Ratio (PSNR) is utilized to assess the presentation of the various strategies. What's more, the Signal-to-Clutter Ratio (SCR) is utilized to assess the exhibition of conservation of solid point focuses of the various strategies. Additionally three other various sorts of quantitative assessment pointers are utilized to assess the presentation of the various channels.For consistent performance, four different noise variation $(\sigma=10,20,40$ and 60$)$ is used. Also, the multi-look images are generated for the synthetic images at four different looks ( $\mathrm{L}=1,2,4$, and 16). Peak Signal-to-Noise Ratio (PSNR): PSNR (Easley et al., 2008) is decibels (dB) defined as

$$
P S N R=20 \log _{10} \frac{255 N}{\|f-\hat{f}\|_{F}}
$$

where $\|.\|_{F}$ is the norm of Frobenius, the image $f$ is of size $\mathrm{N} \times \mathrm{N}$, and $\hat{f}$ denotes the estimated image. Figure 2 shows the noisy synthetic images, and their corresponding denoised images from various despeckling methods. Table 2 quantifies the PSNR measure on every despeckling approach at various speckle level.

From the outcomes, it is demonstrated that the proposed technique can accomplish the most elevated PSNR esteem than the other existing calculations. The perception is that the proposed technique extraordinarily beat LMMSE-PCA and BM3D on most commotion levels legitimizes the proposed denoising model for ASDN. We likewise saw that the presentation of LMMSE-PCA is exceptionally delicate to commotion level variety in logarithmic space. As should be obvious in Table 2, when the clamor level is low, LMMSE-PCA accomplished lower insights than the proposed technique and the equivalent has been portrayed in Figure 2. Nonetheless, with the expansion of commotion level, LMMSE-PCA will in general accomplish tantamount outcomes with our technique as far as PSNR. LMMSE-PCA even accomplished higher PSNR on picture Lena when the clamor level is higher, this is sensible in light of the fact that LMMSE-PCA was intended for AWGN. In the event that clamor level is high, the dot commotion subject to logarithmic activity which is near the Gaussian repetitive sound. Thusly, the strategy can accomplish great outcomes. In any case, when the commotion level is little, spot noise starts to stray from Gaussian dispersion, and its mean worth is not any more zero. This disparity between the exact information and the model suspicion may decrease the productivity of LPG-PCA. Figure 2 delineates the example yields from the proposed despeckling and five other existing strategies with manufactured pictures at commotion change 10 with 16 different looks. As appeared in the figure, pictures by the proposed technique have little antiquities yet a lot of picture subtleties.
Table 2. Performance Analysis of Despeckling Methods with PSNR measure

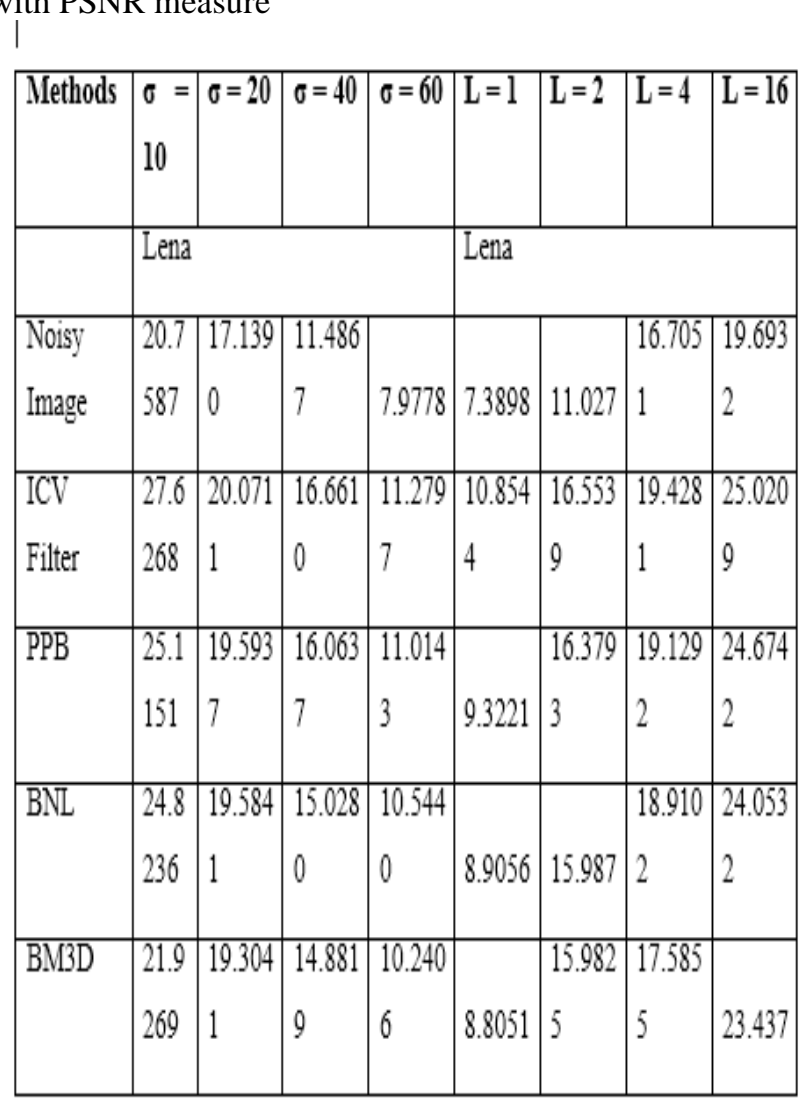

Signal-to-Clutter Ratio (SCR): Clutter is a term used to portray any item that may create undesirable radar returns and which may meddle with typical radar tasks. Mess echoes are arbitrary and have warm commotion like attributes on the grounds that the individual mess parts (scatterers) have irregular stages and amplitudes. By and large, the messiness signal level is a lot higher than the collector clamor level. Subsequently, the radar's capacity to identify targets implanted in high mess foundation relies upon the Signal-to-Clutter Ratio (SCR) as opposed to the SNR. Here the edge pixels are considered as messes (target pixels). SCR can be utilized to assess the exhibition of saving solid point focuses in as SAR picture. SCR is decibels (dB) characterized as (Hou et al., 2012):

$S C R=10 \log _{10} \frac{\sum_{i} \sum_{j}|\hat{s}(i, j)|}{N \sigma_{C}}$

where $\hat{s}(i, j)$ is the point target pixel esteem, $\mathrm{N}$ is the absolute number of point target pixels, and $\sigma_{C}$ is the messiness standard deviation. A huge SCR esteem relates to more readily spot concealment. Table 3 depicts the SCR values received from despeckling methods. Again the proposed method outperforms other methods by attaining the highest SCR value than others for all the synthetic images. 
Table 3. Performance Analysis of Despeckling Methods with SCR measure

\begin{tabular}{|l|l|l|l|l|l|l|l|l|l|}
\hline Methods & $\boldsymbol{\sigma}=\mathbf{1 0}$ & $\boldsymbol{\sigma}=\mathbf{2 0}$ & $\boldsymbol{\sigma}=\mathbf{4 0}$ & $\boldsymbol{\sigma}=\mathbf{6 0}$ & $\mathbf{L}=\mathbf{1}$ & $\mathbf{L = 2}$ & $\mathbf{L = 4}$ & $\mathbf{L}=\mathbf{1 6}$ \\
\hline & Lena & Lena & \multicolumn{6}{|l|}{} \\
\hline $\begin{array}{l}\text { Noisy } \\
\text { Image }\end{array}$ & 3.4991 & 3.1748 & 2.6596 & 2.4265 & 2.4195 & 2.5855 & 2.9135 & 3.1671 \\
\hline $\begin{array}{l}\text { ICV } \\
\text { Filter }\end{array}$ & 3.7693 & 3.4900 & 3.1115 & 2.6014 & 2.5757 & 2.8127 & 3.1197 & 3.7660 \\
\hline PPB & 3.7658 & 3.4512 & 3.0831 & 2.5663 & 2.5548 & 2.7844 & 3.0869 & 3.6444 \\
\hline BNL & 3.6302 & 3.3669 & 3.0612 & 2.5573 & 2.5128 & 2.7411 & 3.0487 & 3.3506 \\
\hline BM3D & 3.6277 & 3.3424 & 2.9463 & 2.4872 & 2.4853 & 2.7251 & 3.0392 & 3.2992 \\
\hline
\end{tabular}

Two more measures are analyzed the preservation of edge quality of the proposed algorithm. The first parameter $\beta$ is used for calculating the edge preservation. It is defined as (Sattar et al., 1996)

$\beta=\frac{\Gamma(\Delta S-\mu(\Delta S), \hat{\Delta S}-\mu(\hat{\Delta S}))}{\sqrt{\Gamma(\Delta S-\mu(\Delta S), \Delta S-\mu(\Delta S)), \Gamma(\widehat{\Delta S}-\mu(\hat{\Delta S}), \hat{\Delta S}-\mu(\hat{\Delta S}))}}$
Where $\Delta S_{\text {and }} \widehat{\Delta S}$ are the filtered high pass of the image $S$ and the image of denoised $\hat{S}$, which is with a size of window of $3 \times 3$ standard estimate of the Laplacian administrator. The over-line administrator speaks to the mean worth, and $\Gamma\left(S_{1}, S_{2}\right)=\sum_{i=1}^{N} S_{1 i *} S_{2 i}$. The connection measure ought to be near solidarity for an ideal impact of edge conservation. Table 4 shows the values of the $\beta$ measure calculated for each denoising methods. The greater value of the proposed method states that this algorithm can preserve the edges better than any other methods.

Table 4. Performance Analysis of Despeckling Methods with $\beta$ measure

\begin{tabular}{|l|l|l|l|l|l|l|l|l|l|l|}
\hline Methods & $\boldsymbol{\sigma}=\mathbf{1 0}$ & $\boldsymbol{\sigma}=\mathbf{2 0}$ & $\boldsymbol{\sigma}=\mathbf{4 0}$ & $\boldsymbol{\sigma}=\mathbf{6 0}$ & $\mathbf{L}=\mathbf{1}$ & $\mathbf{L}=\mathbf{2}$ & $\mathbf{L = 4}$ & $\mathbf{L}=\mathbf{1 6}$ \\
\hline & Lena & \multicolumn{2}{l|}{} \\
\hline $\begin{array}{l}\text { Noisy } \\
\text { Image }\end{array}$ & 0.8906 & 0.7787 & 0.6685 & 0.6005 & 0.6405 & 0.7162 & 0.7962 & 0.9147 \\
\hline $\begin{array}{l}\text { ICV } \\
\text { Filter }\end{array}$ & 0.9595 & 0.8555 & 0.7778 & 0.6683 & 0.7048 & 0.7869 & 0.9082 & 0.9799 \\
\hline PPB & 0.9569 & 0.8403 & 0.7556 & 0.6658 & 0.6820 & 0.7695 & 0.8998 & 0.9724 \\
\hline BNL & 0.9479 & 0.8168 & 0.7455 & 0.6475 & 0.6781 & 0.7562 & 0.8877 & 0.9539 \\
\hline BM3D & 0.9185 & 0.8058 & 0.7074 & 0.6459 & 0.6741 & 0.7531 & 0.8864 & 0.9253 \\
\hline LMIMSE & 0.9171 & 0.7882 & 0.6910 & 0.6361 & 0.6741 & 0.7199 & 0.8455 & 0.9214 \\
\hline $\begin{array}{l}\text { Frost } \\
\text { Filter }\end{array}$ & 0.8998 & 0.7820 & 0.6785 & 0.6164 & 0.6482 & 0.7176 & 0.8119 & 0.9195 \\
\hline
\end{tabular}


Edge-Preserved Index (EPI) is another effective measure to assess the edge protection for genuine SAR picture, which is steady for various kinds of edge pictures and is just influenced by the dot level. The EPI can be represented as (Waske et al., 2007)

$$
E P I=\frac{\sum_{i=1}^{m}\left|I_{D 1}(i)-I_{D 2}(i)\right|}{\sum_{i=1}^{m}\left|I_{O 1}(i)-I_{O 2}(i)\right|}
$$

where $m$ is the pixel number of the chose territory. ID1(i) and ID2(i) speak to the nearby pixel estimations of the despeckled picture along level course. Also, IO1(i) and IO2(i) speak to the contiguous pixel estimations of the first picture. The ideal estimation of EPI is equivalent to one. The EPI is more like one, which means better capacity of edge protection. The normal exhibition of the proposed despeckling technique on manufactured pictures is examined with the clamor change 10 and 20, number of looks 2 and 4. The ICV channel put together denoising is applied with respect to engineered pictures for multiple times at various blends of commotion fluctuation and looks. The ANOVA test is performed to analyze the significance of noise variance and the number of looks, figure 3 depicts the performance comparisons. The summarized ANOVA statistics is depicted in Table 6. The higher $F$ value and the lower $\mathrm{P}$ value represents that the noise variance and the number of looks cause significant changes on denoising performances, for almost all the parameters.

Table5. ANOVA Statistics for the performance of ICV filter

\begin{tabular}{|l|l|l|}
\hline $\begin{array}{l}\text { Performance } \\
\text { Measure }\end{array}$ & F Statistics & P Value \\
\hline PSNR & 48.8709 & $2.90 \mathrm{e}-19$ \\
\hline SCR & 51.5377 & $6.18 \mathrm{e}-20$ \\
\hline B-value & 34.8776 & $2.47 \mathrm{e}-15$ \\
\hline EPI & 56.5709 & $3.78 \mathrm{e}-21$ \\
\hline
\end{tabular}

Table 6 shows the performance of despeckling methods. The outcome hows that the FMM hybrid filter better than the existing approaches.

Table 6. Despeckling Methods Comparison

\begin{tabular}{|l|c|c|c|}
\hline $\begin{array}{l}\text { Filtering } \\
\text { Methods }\end{array}$ & PSNR & SCR & EPI \\
\hline FMM & 29.7254 & 4.7021 & 0.9171 \\
\hline BM3D & 28.2131 & 4.2151 & 0.8122 \\
\hline SWW & 28.1187 & 4.1201 & 0.8005 \\
\hline Frost Filter & 25.5647 & 3.8751 & 0.7517 \\
\hline Mean-Median & 24.3157 & 3.8111 & 0.7721 \\
\hline
\end{tabular}

\section{CONCLUSIONS}

In this paper, a SAR image despeckling plan is proposed dependent on coherence structure based segmentation with half breed filtering approaches. At first, watershed calculation is applied with the edge image to get the homogeneous locales from the SAR image for every area. The cognizant pixels recognized by utilizing Intensity Coherence Vector (ICV) measure, are applied with neighborhood mean filtering. Furthermore, these patches are consolidated to shape total denoised image. Henceforth, the proposed approach doesn't utilize any fixed window size. It considers the coherence pixels for denoising through ICV and edge marker based segmentation just as it protects the limits. The proposed denoising plan is tried on both engineered and genuine SAR images and is contrasted and a few other cutting edge techniques. The outcomes show that the proposed technique is nearly better than referred to strategies both as far as image detail protection and spot clamor decrease. This paper proposes a cross variety channel for SAR despeckling, where the Frost, Median and Mean channels are collected for building a novel hybridization called FMM (Frost-Median-Mean) channel. The SAR pictures are applied with these channels separately consecutively and the result in separated and differentiated and the other denoising procedures. The results show that the proposed FMM channel has pervasive execution than the current channels in the spatial, repeat, hybrid similarly as non-close by territories.

\section{REFERENCES}

[1] Zhou, Z. F., \& Shui, P. L. (2007). Contourlet-based image denoising algorithm using directional windows. Electronics Letters, 43(2), 92-93.

[2] Zhang, L et al (2010). Two-stage image denoising by principal component analysis with local pixel grouping. Pattern Recognition, 43(4), 1531-1549.

[3] Yu, Y et al (2002). Speckle reducing anisotropic diffusion, IEEE Transactions on Image Processing, 11(11), 1260-1270.

[4] $\mathrm{Xu}, \mathrm{L}$ et al (2014). SAR Image Denoising via Clustering-Based Principal Component Analysis. 
[5] Waske, et al (2007). A segment-based speckle filter using multisensoral remote sensing imagery, IEEE Geoscience Remote Sensing Letters, 4(2), 231-235.

[6] Vincent et al (1991). Watersheds in digital spaces: an efficient algorithm based on immersion simulations. IEEE transactions on pattern analysis and machine intelligence, 13(6), 583-598.

[7] Sattar, et al (1996). Image enhancement based on a nonlinear multiscale method. IEEE transactions on image processing, 6(6), 888-895.

[8] Roerdink et al (2000). The watershed transform: Definitions, algorithms and parallelization strategies. Fundamentainformaticae, 41(1), 187-228.

[9] R. Sathish, R. Manikandan, S. Silvia Priscila, B. V. Sara and R. Mahaveerakannan, "A Report on the Impact of Information Technology and Social Media on Covid-19," 2020 3rd International Conference on Intelligent Sustainable Systems (ICISS), Thoothukudi, India, 2020, pp. 224-230, doi: 10.1109/ICISS49785.2020.9316046.

[10] Ranjani, et al (2010). Dual-tree complex wavelet transform based SAR despeckling using interscale dependence. IEEE Transactions on Geoscience and Remote Sensing, 48(6), 2723-2731.

[11] Pass, et al (1997). Comparing images using color coherence vectors, In Proceedings of the fourth ACM international conference on Multimedia, 65-73.

[12] Meyer et al (1990). Morphological segmentation. Journal of visual communication and image representation, 1(1), 21-46.

[13] Lu, Y et al (2013). SAR Image Despeckling with Adaptive Multiscale Products Based on Directionlet Transform. Mathematical Problems in Engineering, 2013.

[14] Lopes, et al. (1990). Adaptive speckle filters and scene heterogeneity, IEEE Transactions on Geoscience and Remote Sensing, 28(6), 992-1000.

[15] Liu, G et al (2013). Structure Preserving SAR image despeckling via L0-minimization. Progress In Electromagnetics Research, 1-22.
[16] Manikandan, $\mathrm{R}$ and Dr.R.Latha (2017). "A literature survey of existing map matching algorithm for navigation technology. International journal of engineering sciences \& research technology", 6(9), 326-331.Retrieved September 15, 2017.

[17] Bianchi, et al (2008). Segmentation-based MAP despeckling of SAR images in the undecimated wavelet domain. Geoscience and Remote Sensing, IEEE Transactions on, 46(9), 2728-2742.

[18] Achim, A et al (2003). SAR image denoising via Bayesian wavelet shrinkage based on heavy-tailed modeling. IEEE Transactions on Geoscience and Remote Sensing, 41(8), 1773-1784. 